\title{
ON A CLASS OF POLYNOMIALLY SOLVABLE TRAVELLING SALESMAN PROBLEMS
}

Abstract. We introduce a class of so-called skew orderable networks. Roughly speaking, to vertices of a skew orderable network $G$ one can assign positive integers in such a way that the assignment, called an ordering function for $G$, reflects certain structural properties of 4-vertex cycles of $G_{\text {. It }}$ is shown that finding a shortest hamiltonian cycle in a skew orderable network is nothing else but constructing an ordering function, which in turn can be obtained in polynomial time with respect to the number of vertices.

1. Introduction. In this paper we consider simple undirected networks (to be called networks), namely $G=(V, E, \varrho)$, where $V$ is the vertex set, $E$ is the set of undirected edges, and $\varrho$ is the weight (or distance) function $\varrho: E \rightarrow \boldsymbol{R}$. In what follows we write $\varrho(v, w)$ instead of $\varrho(\{v, w\})$ for convenience. Moreover, $N_{n}$ denotes the set $\{1,2, \ldots, n\}$ and $|X|$ is the cardinality of the set $X$. A complete $n$-vertex network is denoted by $K_{n}$, while $C_{n}$ is a cycle with $n$ vertices. $H \subseteq G$ means that $H$ is a subnetwork of $G$. A subnetwork of $G$ induced on $X \subseteq V(G)$ is denoted by $G \mid X$.

Let us begin with the following lemma which is fundamental for our approach.

Lemma 1. Let $X=N_{n}$ and let $f: X \times X \rightarrow R$ satisfy the following conditions:

(i) symmetry: $(\forall k, l) f(k, l)=f(l, k)$,

(ii) growth comparability: $(\forall k \leqslant l)(\forall i \leqslant j)(k, l \neq i, j)$

$$
f(l, j)-f(l, i) \geqslant f(k, j)-f(k, i) .
$$

Furthermore, let $S_{X}$ be the symmetric group on $X$ and let $F$ be a function $F: S_{X} \rightarrow R$ defined by

$$
\left(\forall \pi \in S_{X}\right) F(\pi)=\sum_{i=1}^{n-1} f(\pi(i), \pi(i+1))+f(\pi(n), \pi(1)) .
$$


Then $F$ attains its minimal value for

$$
\tilde{\pi}=\left(\begin{array}{ccccccccc}
1 & 2 & 3 & 4 & \ldots & n-3 & n-2 & n-1 & n \\
1 & n-1 & 3 & n-3 & \ldots & 4 & n-2 & 2 & n
\end{array}\right) .
$$

Proof. Induction on $n$. For $n=2,3, F$ is constant, and for $n=4$ it takes at most 3 different values. The permutations to be considered are

$$
\begin{gathered}
\pi_{1}=\left(\begin{array}{llll}
1 & 2 & 3 & 4 \\
1 & 2 & 3 & 4
\end{array}\right), \quad \pi_{2}=\left(\begin{array}{llll}
1 & 2 & 3 & 4 \\
1 & 2 & 4 & 3
\end{array}\right), \\
\tilde{\pi}=\left(\begin{array}{llll}
1 & 2 & 3 & 4 \\
1 & 3 & 2 & 4
\end{array}\right) .
\end{gathered}
$$

We have

$$
\begin{aligned}
& F\left(\pi_{1}\right)-F(\tilde{\pi})=f(3,4)-f(3,1)-f(2,4)+f(2,1), \\
& F\left(\pi_{2}\right)-F(\tilde{\pi})=f(4,3)-f(4,1)-f(2,3)+f(2,1) .
\end{aligned}
$$

By property (ii), both differences are nonnegative. When $n=5$, one should inspect 12 distinct permutations to see that

$$
\left(\begin{array}{lllll}
1 & 2 & 3 & 4 & 5 \\
1 & 4 & 3 & 2 & 5
\end{array}\right)
$$

is the optimal one.

Actually, each permutation is regarded as a cyclic arrangement of elements of $X$. Each pair of the elements neighbouring in such a cycle occurs in one component of $F$. We shall speak of cycles or permutations on $X$ equivalently. The idea of the inductive step is to insert 1 and $n$ properly into the optimal cycle on $\{2,3, \ldots, n-1\}$, showing that the resulting cycle on $X$ is optimal.

Let $Y=X-\{1, n\}$. By assumption and due to symmetry (i),

$$
\tilde{\pi}=\left(\begin{array}{ccccc}
2 & 3 & \ldots & n-2 & n-1 \\
n-1 & 3 & \ldots & n-2 & 2
\end{array}\right) \in S_{Y}
$$

is an optimal cycle on $Y$. Let $\pi$ be any cycle on $Y$ and assume that $i$ and $j$. are neighbours in $\pi$. By $\pi_{j}^{i}$ we denote the cycle on $X$ that is obtained when 1 and $n$ are inserted into $\pi$ between $i$ and $j$ in such a way that 1 becomes a neighbour of $j$ and $n$ a neighbour of $i$, i.e.,

$$
\pi=\left(\begin{array}{llllll}
\ldots & i & j & \ldots
\end{array}\right) \text { and } \pi_{j}^{i}=\left(\begin{array}{llllll}
\ldots & i & n & 1 & j & \ldots
\end{array}\right)
$$

We shall see that

$$
(\forall 2 \leqslant i, j \leqslant n-1)\left(\forall \pi=(\ldots \quad i \quad j \quad \ldots) \in S_{Y}\right) F\left(\pi_{j}^{i}\right) \geqslant F\left(\tilde{\pi}_{n-1}^{2}\right) .
$$


We have

$$
\begin{aligned}
& F\left(\pi_{j}^{i}\right)-F\left(\tilde{\pi}_{n-1}^{2}\right) \\
= & F(\pi)-F(\tilde{\pi})+f(i, n)+f(1, j)-f(i, j)-f(2, n)-f(1, n-1)+f(2, n-1) \\
\geqslant & f(i, n)-f(i, j)-(f(1, n-1)-f(1, j))-f(2, n)+f(2, n-1) \\
\geqslant & f(2, n)-f(2, j)-(f(2, n-1)-f(2, j))-f(2, n)+f(2, n-1)=0 .
\end{aligned}
$$

To consider the situation when 1 and $n$ are inserted into a cycle separately, let us denote by $\pi_{k l}^{i j}$ the cycle on $X$ resulting when 1 is included between $k, l$, and $n$ between $i, j$ in $\pi \in S_{Y}$, with $k, l$ as well as $i, j$ neighbouring in $\pi$. Without loss of generality assume that $i<j$ and $k<l$. Let $l \leqslant j$. Hence

$$
\begin{aligned}
F\left(\pi_{k l}^{i j}\right)- & F\left(\tilde{\pi}_{n-1}^{2}\right) \\
= & F(\pi)-F(\tilde{\pi})+f(i, n)+f(n, j)-f(i, j)+f(k, 1)+f(1, l)-f(k, l) \\
& -f(1, n-1)-f(n, 1)-f(2, n)+f(2, n-1) \\
\geqslant & f(i, n)-f(i, j)-(f(1, n-1)-f(1, l))-(f(k, l)-f(k, 1)) \\
& +f(n, j)+f(2, n-1)-f(n, 1)-f(2, n) \\
\geqslant & f(2, n)-f(2, j)-(f(2, n-1)-f(2, l))-(f(n, l)-f(n, 1)) \\
& +f(n, j)+f(2, n-1)-f(n, 1)-f(2, n) \\
= & f(n, j)-f(n, l)-(f(2, j)-f(2, l)) \geqslant 0 .
\end{aligned}
$$

A similar result is easily obtained for the case $l>j \geqslant k$. Assume finally that $l>k>j>i$. We shall transform $\pi_{k l}^{i j}$ to a certain $\sigma \in S_{X}$ such that

$$
F\left(\pi_{k l}^{i j}\right) \geqslant F(\sigma) \geqslant F\left(\tilde{\pi}_{n-1}^{2}\right) .
$$

This is done as follows. Consider the second neighbour of $i$, say $m$, in $\pi_{k l}^{i j}$. If $m \leqslant k, \sigma$ is obtained by moving the pair $(i n)$ between $k$ and 1 and leaving $m$ and $j$ as neighbours, i.e.,

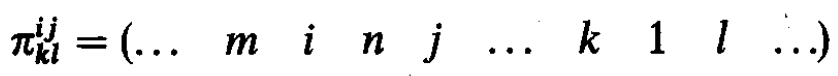

and

$$
\sigma=\left(\begin{array}{lllllllllll} 
& \ldots & m & j & \ldots & k & i & n & 1 & l & \ldots
\end{array}\right)
$$

Hence

$$
\begin{aligned}
F\left(\pi_{k l}^{i j}\right)-F(\sigma) & =f(n, j)-f(n, 1)-(f(m, j)-f(m, i))+f(k, 1)-f(k, i) \\
& \geqslant f(k, j)-f(k, 1)-(f(k, j)-f(k, i))+f(k, 1)-f(k, i)=0 .
\end{aligned}
$$

When $m>k$, to obtain $\sigma$ we exchange simply the places of $i$ and 1 in $\pi_{k l}^{i j}$, i.e.,

$$
\sigma=\left(\begin{array}{lllllllllll}
\ldots & m & 1 & n & j & \ldots & k & i & l & \ldots
\end{array}\right) \text {. }
$$


Again

$$
\begin{aligned}
F\left(\pi_{k l}^{i j}\right)-F(\sigma)= & f(m, i)-f(m, 1)-(f(k, i)-f(k, 1)) \\
& +f(i, n)-f(i, l)-(f(1, n)-f(1, l)) \geqslant 0 .
\end{aligned}
$$

In both cases, 1 and $n$ are neighbours in $\sigma$. Therefore, $\sigma=\psi_{s}^{r}$ for some $\psi \in S_{\mathrm{Y}}$. Since we have already shown that $F\left(\psi_{s}^{\eta}\right) \geqslant F\left(\tilde{\pi}_{n-1}^{2}\right)$, the proof is completed.

Our approach works as follows. Given a network $G=(V, E, \varrho)$ we shall try to construct an injective mapping $\omega: V \rightarrow N_{n}$ such that a function defined by

$$
f(i, j)=\varrho\left(\omega^{-1}(i), \omega^{-1}(j)\right)
$$

has property (ii) of Lemma 1 . Symmetry (i) is obviously satisfied since $G$ is undirected. Now, since $F(\pi)$ defined by (1) gives the length of the cycle

$$
\left(\omega^{-1}(\pi(1)), \omega^{-1}(\pi(2)), \ldots, \omega^{-1}(\pi(n)), \omega^{-1}(\pi(1))\right)
$$

in $G$, the shortest hamiltonian tour in $G$ is determined by (3) $\left(\omega^{-1}(1), \omega^{-1}(n-1), \omega^{-1}(3), \ldots, \omega^{-1}(n-2), \omega^{-1}(2), \omega^{-1}(n), \omega^{-1}(1)\right)$, and hence the TSP for $G$ has been reduced to the problem of constructing an appropriate $\omega$.

For other tractable cases of the TSP the reader is referred to [1].

2. Orderable networks and their properties. Let us consider first a simpler case where the function $f$ in Lemma 1 has an additional property

$$
(\forall k)(\forall i \leqslant j) f(k, j)-f(k, i) \geqslant 0 .
$$

Using (2) one arrives at the following

Defintrion 1. A network $G=(V, E, \varrho)$, where $|V|=n$, is called orderable iff there exists an injective function $\omega: V \rightarrow N_{n}$ such that

$$
(\forall x, y, z \in V) \varrho(x, y)<\varrho(x, z) \Rightarrow \omega(y)<\omega(z) .
$$

$\omega$ is called an ordering function for $G$. Let $\Omega(G)$ denote the set of all ordering functions for $G$, and Ord denote the class of all orderable networks. If a particular $\omega \in \Omega(G)$ is selected, $G$ is said to be $\omega$-ordered.

Example 1. $G \in \operatorname{Ord} \Rightarrow(\forall H \subseteq G) H \in$ Ord. However, orderability of all proper subnetworks is not sufficient for the orderability of the whole network, in general. An example is a cycle $C_{4}$ (Fig. 1). One can verify that

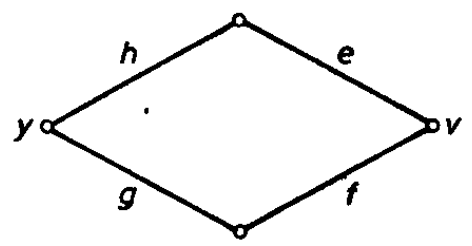

Fig. 1 
$C_{4} \in$ Ord iff $e_{\max } \cap e_{\min }=\varnothing$, where $e_{\max }$ and $e_{\min }$ denote the longest and the shortest edges of $C_{4}$, respectively. On the other hand, the deletion of a single edge results in an orderable subnetwork of $C_{4}$.

Definition 2. Let $G=(V, E, \varrho)$. Then $\vec{G}=\left(V^{\prime}, E^{\prime}\right)$, called the associate of $G$, is a digraph defined on the basis of $G$ as follows:

$$
\begin{array}{ll}
1^{\circ} & V^{\prime}=V, \\
2^{\circ} & E^{\prime}=\{(u, v) \in V \times V:(\exists x \in V) \varrho(x, u)>\varrho(x, v)\} .
\end{array}
$$

The vertex $x$ appearing in $2^{\circ}$ is said to generate the arc $(u, v)$.

TheOREM 1. $G \in$ Ord iff $\vec{G}$ contains no directed cycles.

Proof: Suppose that $\vec{G}$ contains a cycle $C$ and let $u$ and $v$ be any two vertices of $C$. Thus both $(u, v)$ and $(v, u)$ appear in the transitive closure $\vec{G}^{\text {tc }}$ of $G$. Since

$$
(u, v) \in E\left(\vec{G}^{\mathrm{t}}\right) \quad \text { iff } \quad(\forall \omega \in \Omega(G)) \omega(u)>\omega(v),
$$

we conclude that $G \notin$ Ord.

Now, let $\vec{G}$ be acyclic. This means that all induced subdigraphs of $\vec{G}$ contain a vertex $v$ with outdeg $(v)=0$, because otherwise $\vec{G}$ would contain a cycle. We shall construct $\omega \in \Omega(G)$. Let $v$ be any vertex of $\vec{G}$ with outdeg $(v)$ $=0$. Set $\omega(v)=1$ and remove $v$ from $\vec{G}$. Next, in $\vec{G}_{v}=\vec{G} \mid(V-\{v\})$ we find a vertex $u$ such that outdeg $(u)=0$ and we set $\omega(u)=2$, removing $u$ from $\vec{G}_{v}$, and so on. It is now easy to see that $G$ is $\omega$-ordered, and thus $G \in$ Ord.

Let us estimate the complexity of constructing $\omega$ for $G$. Since we have to consider all pairs of incident edges in $G$ in order to obtain the associate $\vec{G}$, this requires

$$
\left.\sum_{i=1}^{n}\left(\begin{array}{c}
\operatorname{deg}\left(v_{i}\right) \\
2
\end{array}\right) \leqslant \frac{n(n-1)(n-2)}{2} \text { (or }<2 m^{2}-m\right)
$$

steps, where $n=|V(G)|$ and $m=|E(G)|$. The algorithm for constructing $\omega \in \Omega(G)$ given in the proof of Theorem 1 requires no more than $O\left(n^{2}\right)$ steps for completion, since any time a vertex is removed from $\vec{G}$, one has to decrease the valences of at most all remaining vertices. Thus the total complexity of the method is not greater than $O\left(n^{3}\right)$.

One can easily establish several results concerning orderability of various network classes, e.g., orderability of all acyclic networks and of all odd cycles. Our aim, however, is to study in detail the orderability of complete and, in general, hamiltonian networks and to employ additional restrictive conditions which will yield property (ii) of $f$ in Lemma 1 .

Theorem 2. $K_{n} \in$ Ord $^{\circ}$ iff $\left(\forall C_{4} \subseteq K_{n}\right) C_{4} \in$ Ord.

Proof. If $K_{n}$ is orderable, then each of its subnetworks is orderable (Example 1). To prove the converse implication, observe that a nonorderable $C_{4}$ is contained in $G$ iff there exists a directed cycle of length 2 in $\vec{G}$. Assuming $K_{n} \notin$ Ord, we shall see that $C_{2} \subseteq \vec{K}_{n}$. Thus, let $r$ be the length of 
the shortest cycle

$$
C_{r}=\left(v_{1} \rightarrow v_{2} \rightarrow \ldots \rightarrow v_{r} \rightarrow v_{1}\right)
$$

contained in $\vec{K}_{n}, 2<r \leqslant n$. If $r<n$, then for every $v \in V\left(K_{n}\right)-\left\{v_{1}, \ldots, v_{r}\right\}$ the following inequalities should hold (cf. Fig. 2):

$$
\varrho\left(e_{1}\right) \geqslant \varrho\left(e_{2}\right) \geqslant \varrho\left(e_{3}\right) \geqslant \ldots \geqslant \varrho\left(e_{r}\right) \geqslant \varrho\left(e_{1}\right) .
$$

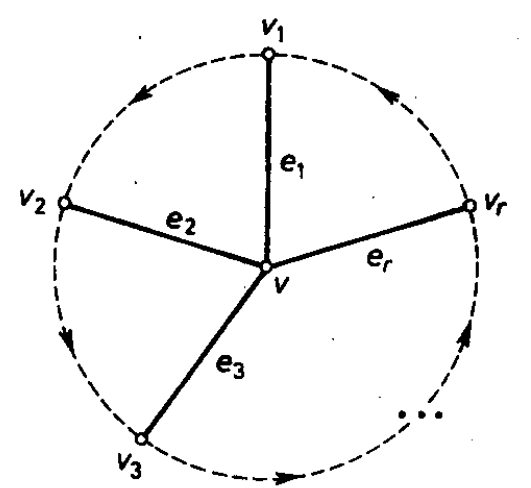

Fig. 2

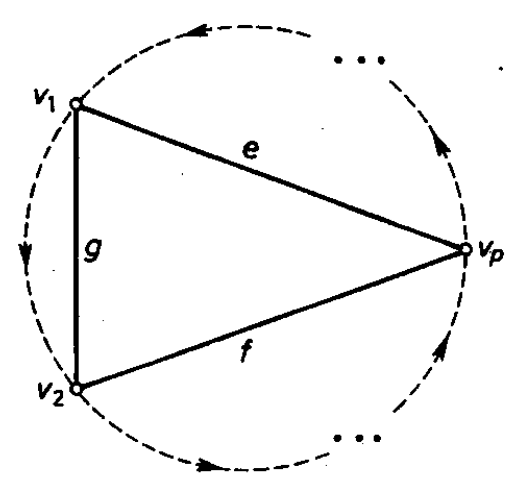

Fig. 3

However, if any of the inequalities were strong, we would have a contradiction $\varrho\left(e_{1}\right)>\varrho\left(e_{1}\right)$. Therefore, no arc of $C_{r}$ is generated by an "external" vertex and we can consider both cases $r<n$ and $r=n$ uniformly.

Let $v_{p}(3 \leqslant p \leqslant r)$ be a vertex that generates $\left(v_{1}, v_{2}\right)$ (Fig. 3). We have $\varrho(e)>\varrho(f)$. By assumption, $C_{r}$ is the shortest cycle in $\vec{K}_{n}$, and hence neither $\left(v_{1}, v_{p}\right)$ nor $\left(v_{p}, v_{2}\right)$ belongs to $E\left(\vec{K}_{n}\right)$. But this means that $\varrho(f) \geqslant \varrho(g) \geqslant \varrho(e)$, which contradicts $\varrho(e)>\varrho(f)$. We conclude that the number $r$, if greater than 2 , can always be reduced.

Finally, let us formulate a condition related to (ii) in Lemma 1. Consider a cycle $C_{4}$, with

$$
E\left(C_{4}\right)=\{e, f, g, h\}, \quad \text { where } \varrho(e) \leqslant \varrho(f) \leqslant \varrho(g) \leqslant \varrho(h) .
$$

The cycle is called skew iff

$$
\varrho(e)+\varrho(h) \geqslant \varrho(f)+\varrho(g) .
$$

A network is called skew iff all its cycles $C_{4}$ are skew. Now one can verify that the combination of skewness and orderability makes Lemma 1 applicable as far as the network is complete (!), since one must be sure that all edges of (3) do exist in $G$. The next section will be devoted to the study of noncomplete hamiltonian networks.

It should be noted that both skewness and orderability have no relation to the triangle inequality, which may hold or not in a skew network $G \in$ Ord.

3. Strong orderability. The usual way of handling the TSP in noncomplete networks is to supply "very long" edges for the lacking ones. These 
additional edges will never be selected for constructing the shortest cycle because of their length, provided that the original network is hamiltonian itself. Let us denote by $G^{c}$ a completion of $G=(V, E, \varrho)$, where for supplied edges $f \notin E$ we put

$$
\varrho(f)>\sum_{e \in E}|\varrho(e)|
$$

If $\varrho$ is a set constant on supplementary edges, we write $G^{\text {cs }}$ for the completion. Note that $G^{\text {cs }}$ is not necessarily orderable even if $G$ is so.

Definition 3. $G$ is called strongly orderable iff $G^{\text {es }} \in$ Ord.

LEMMA 2. $G$ is strongly orderable iff for every $U \subseteq V(G),|U|=4, G \mid U$ is either complete orderable and discrete (i.e., has no edges) or has one of the forms shown in Fig. 4, where

$$
\varrho(a) \leqslant \varrho(b) \leqslant \varrho(c) \leqslant \varrho(e) \quad \text { and } \quad \varrho(b) \leqslant \varrho(d) \leqslant \varrho(e) .
$$
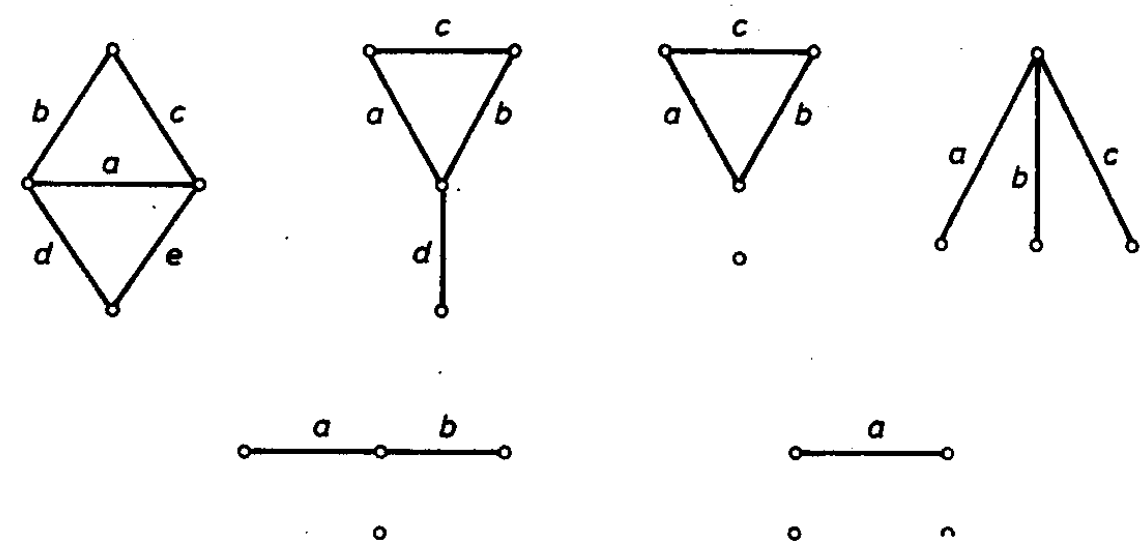

Fig. 4

Proof. By Theorem 2, $G^{\text {cs }} \in$ Ord iff it contains no nonorderable $C_{4}$. Consider any 4-clique $K_{4}$ in $G^{\text {cs }}$ (Fig. 5). Let $\omega \in \Omega\left(G^{\text {cs }}\right)$ and

$$
\omega(v) \leqslant \omega(x) \leqslant \omega(y) \leqslant \omega(z) .
$$

From (5) it follows that

$$
\varrho(a) \leqslant \varrho(b) \leqslant \varrho(c) \leqslant \varrho(e) \leqslant \varrho(f) \quad \text { and } \quad \varrho(b) \leqslant \varrho(d) \leqslant \varrho(e) .
$$

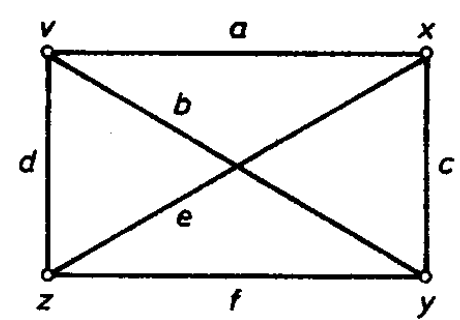

Fig. 5

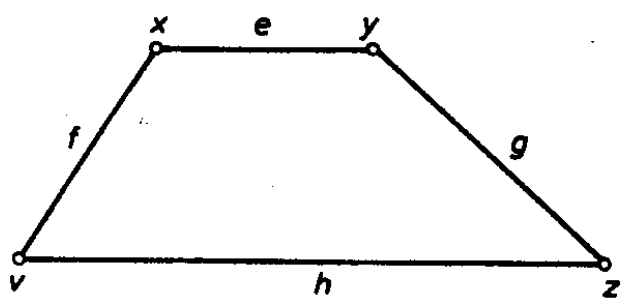

Fig. 6 
Possible forms of $G \mid\{v, x, y, z\}$ are now obtained by stepwise deletion of the longest edges in $K_{4}$. On the other hand, whenever "long" edges are added to any graph in Fig. 4, no nonorderable $C_{4}$ occurs.

The problem we are faced with now is to provide that (6) will not be violated in a completion $G^{\mathrm{c}}$ of a skew and strongly orderable network $G$. As we shall see, it can always be done by varying the lengths of supplementary edges in $G^{\mathrm{cs}}$.

LemMa 3. For every skew and strongly orderable network $G=(V, E, \varrho)$ there exists a skew and orderable completion $G^{\mathrm{c}}$.

Proof. Set

$$
M=2+\sum_{a \in E}|\varrho(a)| .
$$

For every $\{x, y\} \notin E$ put $\varrho(x, y)=M$. The completion $G^{\text {cs }}$ obtained so far is orderable since $G$ is strongly orderable. Now select any $\omega \in \Omega\left(G^{\text {cs }}\right)$ and for every supplementary edge $\{x, y\}$ set

$$
\varrho(x, y)=M^{\omega(x)+\omega(y)} \text {. }
$$

Observe that $G^{\mathrm{c}}$ just constructed is still $\omega$-ordered. We claim that (6) is satisfied for every $C_{4}$ contained in $G^{\mathrm{c}}$. We shall show that this is the case for the worst possible pattern of $C_{4}$, i.e., such that (Fig. 6) $f, g$ and $h$ are supplementary edges and $\varrho(e)$ may be either negative or positive. It follows that $\omega(x)<\omega(z)$ and $\omega(y)<\omega(v)$. We have

$$
\varrho(f)=M^{\omega(x)+\omega(v)}, \quad \varrho(g)=M^{\omega(y)+\omega(z)} \quad \text { and } \quad \varrho(h)=M^{\omega(v)+\omega(z)} .
$$

Hence

$$
\begin{aligned}
M^{\omega(v)+\omega(z)}+\varrho(e) & =\left(2+\sum_{a \in E}|\varrho(a)|\right) M^{\omega(v)+\omega(z)-1}+\varrho(e) \\
& \geqslant 2 M^{\omega(v)+\omega(z)-1}+\sum_{a \in E}|\varrho(a)|+\varrho(e) \geqslant M^{\omega(x)+\omega(x)}+M^{\omega(y)+\omega(z)}
\end{aligned}
$$
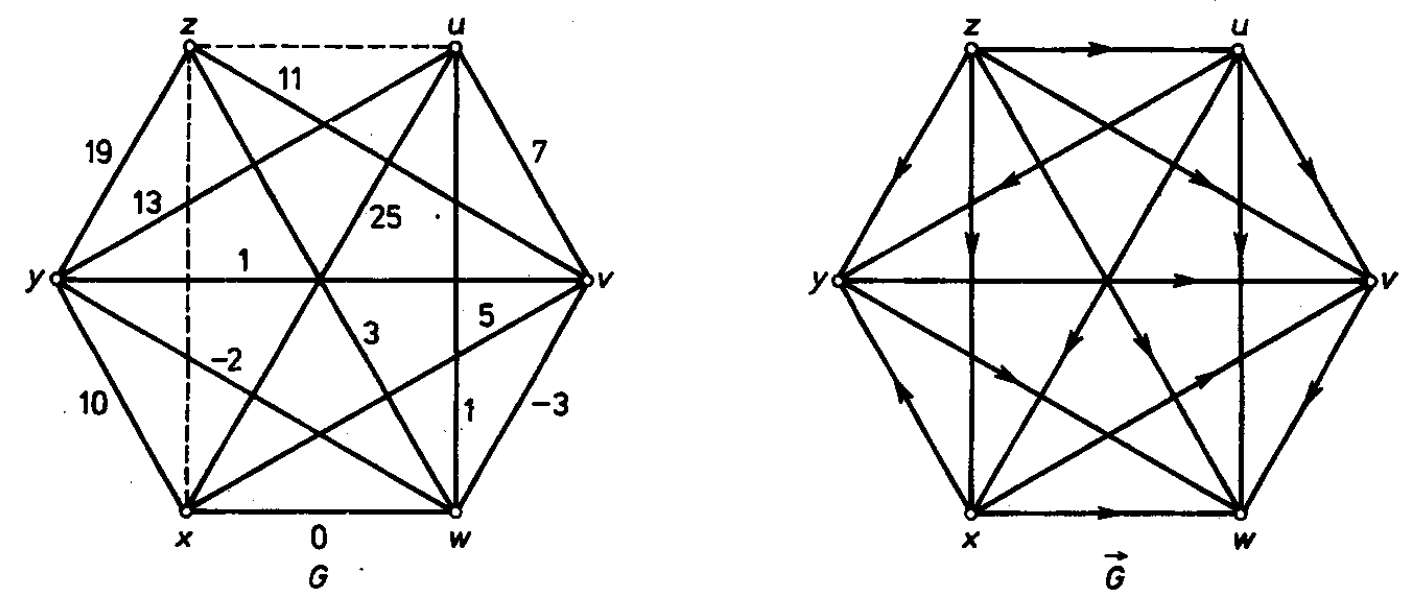

Fig. 7 
It has to be realized that Lemma 3 is only of theoretical importance and not of practical one. Lemma 1 (ii) requires the whole orderable network to be skew and $G^{\text {cs }}$ need not be such. In spite of this, (3) works properly on $G^{\text {cs }}$ (provided $G$ is hamiltonian) since $\omega$ is not affected in any way while $G^{\text {cs }}$ is being "strained" to the skew $G^{\mathrm{c}}$.

To sum up, our method applies to hamiltonian networks that are both skew and strongly orderable. When a generalized TSP is to be considered for nonhamiltonian networks, one needs a different approach in which supplementary edges represent the shortest paths between nonadjacent vertices.

Example 2. Consider a skew network $G \in$ Ord shown in Fig. 7 (solid lines). After supplying the edges $\{z, x\}$ and $\{z, u\}$ with the weight

$$
M=\sum_{e \in E}|\varrho(e)|=100
$$

we find $\vec{G}$, the associate of $G($ Fig. 7). Next $\omega \in \Omega(G)$ is computed: $\omega(w)=1$, $\omega(v)=2, \omega(y)=3, \omega(x)=4, \omega(u)=5$ and $\omega(z)=6$. Now (3) can be applied and we have

$$
\begin{aligned}
C & =\left(\omega^{-1}(1), \omega^{-1}(5), \omega^{-1}(3), \omega^{-1}(4), \omega^{-1}(2), \omega^{-1}(6), \omega^{-1}(1)\right) \\
& =(w, u, y, x, v, z, w) .
\end{aligned}
$$

The length of $C$, the shortest hamiltonian cycle in $G$, is 43 .

In the general case, $\omega \in \Omega(G)$ is constructed without assumption (4). Property (ii) of Lemma 1 together with (2) yield the following constraint:

$$
\begin{aligned}
(\forall u, v, x, y \in V(G)) \varrho(u, x)-\varrho(u, y)> & \varrho(v, x)-\varrho(v, y), \\
& \omega(u)>\omega(v) \text { or } \omega(y)>\omega(x) .
\end{aligned}
$$

The construction of $\omega \in \Omega(G)$ is a bit more complicated then, since the alternative in (7) causes some new difficulties. This case will be discussed elsewhere.

Acknowledgement. The author is greatly indebted to Prof. M. M. Sysło for valuable discussion and remarks.

\section{Reference}

[1] P. C. Gilmore, E. L. Lawler and D. B. Shmoys, Well-solved special cases [of the TSP], pp. 87-144 in: E. L. Lawler, J. K. Lenstra, A. H. G. Rinnooy Kan and D. B. Shmoys (eds.), The Traveling Salesman Problem, J. Wiley, 1985.

\footnotetext{
INSTITUTE OF PHYSICS

NICOLAUS COPERNICUS UNIVERSITY

87-100 TORUŃ
} 